\title{
The Clinical Future of Repetitive Transcranial Magnetic Stimulation and Depression: Separating Hope From Hype
}

\author{
Stefano Pallanti, MD, PhD, Thomas E. Schlaepfer, MD, and Sarah H. Lisanby, MD
}

One of the key messages of the Sequenced Treatment Alternatives to Relieve Depression study ${ }^{1}$ was that only one third of patients with a diagnosis of major depressive disorder (MDD) achieve remission after the first treatment with an antidepressant. Management of most patients after one or more failed trials moves beyond the currently available evidence base and therefore represents a significant clinical challenge. Over the course of the four treatment steps, the cumulative remission rate was $67 \%$ and remission was more likely to occur during the first two treatment levels $(20 \%$ to $30 \%)$ rather than during levels 3 and 4 (10\% to $20 \%)$. These finding suggest that after two consecutive unsuccessful antidepressant trials a change in pharmacologic mode of action has a low probability to affect the likelihood of remission from MDD. The switch to a third antidepressant treatment resulted in an even lower remission rate than the one achieved in the first two levels. Furthermore, the discontinuation rates due to treatment intolerance was shown to rise across sequenced levels. At this point, the search for effective and tolerable alternative options to pharmacotherapy is essential.

Some have placed hope that repetitive transcranial magnetic stimulation (rTMS) will help to meet this need, because other alternatives are either ineffective or too invasive. Given its welldocumented efficacy profile, electroconvulsive therapy (ECT) is the main treatment option for patients who do not respond to medication and psychotherapy. While modern forms of ECT have dramatically improved its side-effect profile, the risk of cognitive side effects still represents a barrier to treatment, motivating the development of alternatives with fewer side effects (eg, magnetic seizure therapy). Vagus nerve stimulation $n^{2,3}$ is Food and Drug Administration-approved for medication resistant chronic depression, but its remission rates fall short of that seen with ECT, and its availability has been limited due to expense. Deep brain stimulation holds great promise, even in ECT-resistant cases, but it is still at the experimental stage for the depression indication and carries a higher degree of invasiveness.

TMS was introduced to neurophysiology in $1985 .{ }^{4}$ The principle of TMS is based on Faraday's law of electromagnetic induction. Electrical current flowing through a coil induces a magnetic field (Ampere's Law). Scalp and skull are transparent to magnetic fields. When it reaches the brain, the pulsating magnetic field induces an electrical current, resulting in neuronal depo-

Dr. Pallanti is associate professor of psychiatry at the University of Florence in Italy and adjunct professor at Mount Sinai School of Medicine in New York City. Dr. Schlaepfer is vice chair and professor of psychiatry and psychotherapy at University of Bonn in Germany and associate professor of psychiatry and mental health at the Johns Hopkins University in Baltimore, Maryland. Dr. Lisanby is professor of clincal psychiatry and director of the Brain Stimulation Division at Columbia University/New York State Psychiatric Institute in New York City.

Faculty Disclosures: Dr. Pallanti has received honoraria from Eli Lilly, Pfizer, Solvay, and Transcept; and has received minister grants for TMS in anxiety research support. Dr. Schlaepfer is chair of the World Federation of Societies of Biological Psychiatry (WFSBP) task force on Brain Stimulation and co-chair of the WFSBP task force on ECT. Dr. Lisanby chairs the APA task force on ECT and has received research support from Advanced Neuromodulation Systems/Saint Jude, Brainsway, Cyberonics, Fisher, Magstim, Magventures, and Neuronetics. She is co-inventor on a patent application filed by Columbia University for TMS technology.

Please direct all correspondence to: Stefano Pallanti, MD, PhD, Visiting Associate Professor, Department of Psychiatry, Mount Sinai School of Medicine, One Gustave L. Levy Place, Box 1230, New York, NY 10029. Email: stefano.pallanti@mssm.edu. 
larization in the cortical tissue underlying the coil. Different from other stimulation methods, TMS allows for a more localized, spatially limited stimulation achieved noninvasively.

rTMS is capable of enhancing or decreasing cortical excitability of that area of interest beyond the duration of stimulation trains. Studies on the motor cortex have revealed that $1 \mathrm{~Hz}$ of stimulation has a largely suppressive effect while frequencies of $\geq 10 \mathrm{~Hz}$ are largely excitatory. ${ }^{5,6}$ Due to its ability to modulate cortical excitability, rTMS have been tested as a therapeutic tool in several neuropsychiatric disorders. To date, the best clinical effects have been observed using rTMS for the treatment of resistant MDD. Research on rTMS for the treatment of patients with depression has been conducted now for a period of $>15$ years. One meta-analysis reported a mean weighted effect size (calculated as mean differences between sham and rTMS of the pre-treatment post-treatment change in rating scales) in the treatment of MDD of 0.55. ECT was superior to rTMS in the treatment of depression (mean weighted effect size -0.47 ), but rTMS has substantially fewer side effects. ${ }^{7}$

The safety profile of TMS has been studied extensively and it is considered generally safe and very well tolerated. A recent paper on the safety and tolerability of rapidly pulsed TMS in MDD, using quite high stimulation parameters on the left dorsolateral prefrontal cortex (DLPFC) for a period of 12 weeks, reported a discontinuation rate of $8 \%$, significantly lower than the $37 \%$ reported in controlled trial of standard antidepressant. ${ }^{8}$ These data strongly support the view that rTMS can be safely administrated in an outpatient setting. The most common side effect is short lasting headache and scalp discomfort. However, rTMS carries a risk seizure, especially when applied with specific parameters such as high frequency and short duration of off time between trains. ${ }^{9,10}$ An adequate pre-treatment clinical screening for potential seizure risk, and adherence to published safety guidelines governing the selection of stimulation parameters, reduce but do not eliminate this risk. Given the good tolerability of rTMS, it may constitute an alternative to medication in particular populations where one would like to avoid the side effects of medications, such as in the elderly, medical comorbidities, and pregnancy. However, few studies have examined the safety of TMS in the context of medical cormor- bidities, fewer still have examined the safety in pregnancy, and a handful of studies suggest efficacy may be lower in the aged. Future research should address safety and efficacy in these and other special populations.

Another possible clinical application of rTMS is as augmentation strategy to antidepressant. Some studies investigated the effectiveness of rTMS in combination with antidepressants in non-drug-resistant MDD patients. It has been proposed that antidepressants and rTMS may share some common mechanism of action and target some common regions in the brain, but little is known about their possible interaction." A recent sham-controlled trial showed that active rTMS shortened the time to response in treatment with venlafaxine, escitalopram, and sertraline and this effect was statistically significant from the first week of treatment to the fourth week, while at the end of the follow-up it showed a trend toward significance both in average improvement on the Hamilton Rating Scale for Depression and in remission rates. ${ }^{12}$ Considering its benign side-effects profile of rTMS, it might be developed into an interesting augmentation strategy. However, it should be noted that the safety guidelines for TMS dosing were developed in a small group of healthy volunteers who were medication free. The effect of concomitant medications on seizure risk with rTMS, and on other potential interactions between TMS and medication effects needs to be carefully studied.

rTMS might also constitute a cost-effective treatment for patients who have failed to receive sufficient benefit from initial antidepressant pharmacotherapy, which is actually where it is currently FDA indicated (for adults who have failed to respond to a single adequately dosed antidepressant medication). When used at earlier levels of treatment resistance, significant cost savings may be expected relative to the current standard of care, and this is also where studies report the best efficacy. ${ }^{13}$ The incremental cost-effectiveness and the direct cost burden compared with sham treatment were estimated, and compared with the current standard of care. In a recent study, the cost effectiveness of rTMS was described using an incremental cost-effectiveness ratio (ICER) per quality-adjusted life year (QALY). Compared with sham treatment and at a cost of $\$ 300$ per treatment session, TMS provides an ICER of $\$ 34,999$ per QALY, which is "willingness to pay" standard of $\$ 50,000$ per 
QALY for a new treatment for major depression. ${ }^{14}$ When productivity gains due to clinical recovery were included, the ICER was reduced to $\$ 6,667$ per QALY. In open-label conditions, TMS provided a net cost saving of $\$ 1,123$ per QALY when compared with the current standard of care. In the open-label condition, cost savings increased further when the costs for productivity losses were included in the model (net savings $\$ 7,621$ ). The major benefits of treating MDD using rTMS were greater in those patients at the earliest levels of treatment resistance.

But let us pause for a moment to consider whether rTMS can truly live up to this hope of offering safe and effective alternatives where medication leaves off. Despite the potential utility of rTMS and the advantages that it has compared to other stimulation techniques, several important questions remain unanswered about the best clinical use of this treatment. The optimal methods for administration of rTMS remain unclear and considerable research remains to be done to define these variables. Optimizing the efficacy of rTMS will be key to whether it will play a viable clinical role in the treatment of depression, given that much more effective alternatives are available (eg, ECT). The literature suggests that different rTMS parameters (cortical target, frequency and intensity of stimulation, compound frequencies such as theta burst, ${ }^{15,16}$ and novel coil shapes ${ }^{17}$ ) can influence outcome. Studies suggest that high-frequency rTMS over the DLPFC or slow-frequency rTMS over the right DLPFC are equivalent in efficacy. ${ }^{18,19}$ The rationale might be that restoring the balance between left and right prefrontal cortex activity is in fact more important than establishing absolute increases or decreases in activity per se. However, the vast majority of the trials conducted into the technique have evaluated the efficacy of high-frequency rTMS applied to the left DLPFC. ${ }^{20}$ It is this dosage of rTMS that, at the end of 2008, received clearance by the United States FDA for the treatment of adults with unipolar MDD who had failed to achieve satisfactory response to one antidepressant medication given at an adequate dosage and duration. Whether sequential bilateral application of slow-frequency rTMS over the right DLPFC followed by high-frequency TMS over left DLPFC, or whether low frequency on the right alone will ultimately be more effective awaits further evaluation. ${ }^{21,22}$

Suitable patients for the application of rTMS according to FDA criteria must have failed one previous antidepressant trial. ${ }^{23}$ However, it remains unclear which factors are predictive of response to rTMS. Future research should focus on sociodemographic predictors, comorbidity, and clinical predictors of response to rTMS. Different factors may also differentiate the likelihood of response to one set of parameters versus another one.

It is also important to take in consideration the procedure for localizing the target areas. Usually the depression target is located by moving the coil $5 \mathrm{~cm}$ anterior to the scalp location that elicits a twitch in the contralateral hand muscle. However, recent work suggests that structural and functional image guidance can improve effect sizes with rTMS, which is expected given its relative focality. While availability of frameless neuronavigation in the clinical setting may be limited by cost, the cost of suboptimal treatment should be borne in mind as well.

\section{CONCLUSION}

Evidence supports the relative safety of rTMS, and FDA clearance has made it clinically available. However, many unanswered questions remain, the answers to which will be vital in determining the clinical future of rTMS. These questions include: What is the best dosage? How can we optimize efficacy? How is it best used in combination with medications? What are the predictors of good response? Can it be made safe and effective in special clinical populations that have the most need for an alternative to medications? While the acute efficacy of rTMS is clearly lower than that of ECT, its safety may make it an interesting alternative for a subset of patients who for selected reasons may not be suitable candidates for treatment with antidepressants.

Having rTMS as a new tool in the clinical toolbox is indeed an important development for our field that offers new hope for our patients. Yet, we owe it to our patients to learn the proper and most effective use of this tool that the hope does not become lost in the hype. CNS

\section{REFERENCES}

1. Rush AJ, Fava M, Wisniewski SR, et al. Sequenced treatment alternatives to relieve depression (STAR*D): rationale and design. Control Clin Trials. 2004;25(1):119-142.

2. Kosel M, Schlaepfer TE. Beyond the treatment of epilepsy: New applications of vagus nerve stimulation (VNS) in psychiatry. CNS Spectr. 2003;8(7):515-521.

3. Schlaepfer T, Frick C, Zobel A, et al. Vagus nerve stimulation for depression: efficacy and safety in a European study. Psychol Med. 2008;38:651-662.

4. Barker AT, Jalinous R, Freeston IL. Non-invasive magnetic stimulation of human 
motor cortex. Lancet. 1985;1(8437):1106-1107.

5. Chen R, Classen J, Gerloff C, et al. Depression of motor cortex excitability by lowfrequency transcranial magnetic stimulation. Neurology. 1997;48(5):1398-1403.

6. Pascual-Leone A, Tormos JM, Keenan J, Tarazona F, Canete C, Catala MD. Study and modulation of human cortical excitability with transcranial magnetic stimulation. J Clin Neurophysiol. 1998;15(4):333-343.

7. Slotema CW, Blom JD, Hoek HW, Sommer IE. Should we expand the toolbox of psychiatric treatment methods to include repetitive transcranial magnetic stimulation (rTMS)? a meta-analysis of the efficacy of rTMS in psychiatric disorders. J Clin Psychiatry. 2010;71(7):873-874.

8. Janicak PG, O'Reardon JP, Sampson SM, et al. Transcranial magnetic stimulation in the treatment of major depressive disorder: a comprehensive summary of safety experience from acute exposure, extended exposure, and during reintroduction treatment. J Clin Psychiatry. 2008;69(2):222-232.

9. Chen R, Gerloff C, Classen J, Wassermann EM, Hallett M, Cohen LG. Safety of different inter-trains intervals for repetitive transcranial magnetic stimulation and recommendations for safe ranges of stimulation paremeters. Electroencephalogr Clin Neurophysiol. 1997;105:415-421.

10. Tharayil BS, Gangadhar BN, Thirthalli J, Anand L. Seizure with single-pulse transcranial magnetic stimulation in a 35-year-old otherwise-healthy patient with bipolar disorder. J Ect. 2005;21(3):188-189.

11. Brunoni AR, Fraguas R, Fregni F. Pharmacological and combined interventions for the acute depressive episode: focus on efficacy and tolerability. Ther Clin Risk Manag. 2009;5:897-910.

12. Rossini D, Magri L, Lucca A, Giordani S, Smeraldi E, Zanardi R. Does rTMS hasten the response to escitalopram, sertraline, or venlafaxine in patients with major depressive disorder? A double-blind, randomized, sham-controlled trial. J Clin Psychiatry. 2005;66(12):1569-1575.

13. Lisanby SH, Husain MM, Rosenquist PB, et al. Daily left prefrontal repetitive transcranial magnetic stimulation in the acute treatment of major depression: clinical predictors of outcome in a multisite, randomized controlled clinical trial. Neuropsychopharmacol. 2009;34:522-534.
14. Simpson KN, Welch MJ, Kozel FA, Demitrack MA, Nahas Z. Cost-effectiveness of transcranial magnetic stimulation in the treatment of major depression: a health economics analysis. Adv Ther. 2009;26(3):346-368.

15. Grossheinrich N, Rau A, Pogarell 0, et al. Theta burst stimulation of the prefrontal cortex: safety and impact on cognition, mood, and resting electroencephalogram. Biol Psychiatry. 2009;65(9):778-784

16. Chistyakov AV, Rubicsek O, Kaplan B, Zaaroor M, Klein E. Safety, tolerability and preliminary evidence for antidepressant efficacy of theta-burst transcranial magnetic stimulation in patients with major depression. Int J Neuropsychopharmacol. 2010;13(3):387-393.

17. Levkovitz. Deep transcranial magnetic stimulation over the prefrontal cortex: evaluation of antidepressant and cognitive effects in depressive patients. Brain Stim. 2009;2:188-200.

18. Stern WM, Tormos JM, Press DZ, Pearlman C, Pascual-Leone A. Antidepressant effects of high and low frequency repetitive transcranial magnetic stimulation to the dorsolateral prefrontal cortex: a double-blind, randomized, placebo-controlled trial. J Neuropsychiatry Clin Neurosci. 2007;19(2):179-186.

19. Fitzgerald PB, Hoy K, Daskalakis ZJ, Kulkarni J. A randomized trial of the antidepressant effects of low- and high-frequency transcranial magnetic stimulation in treatment-resistant depression. Depress Anxiety. 2009;26(3):229-234

20. O'Reardon JP, Solvason HB, Janicak PG, et al. Efficacy and safety of transcranial magnetic stimulation in the acute treatment of major depression: a multisite randomized controlled trial. Biol Psychiatry. 2007;62(11):1208-1216

21. Fitzgerald PB, Benitez J, de Castella A, Daskalakis ZJ, Brown TL, Kulkarni J. A randomized, controlled trial of sequential bilateral repetitive transcranial magnetic stimulation for treatment-resistant depression. Am J Psychiatry. 2006;163(1):88-94.

22. Pallanti S, Bernardi S, Di Rollo A, Antonini S, Quercioli L. Unilateral low frequency versus sequential bilateral repetitive transcranial magnetic stimulation: is simpler better for treatment of resistant depression? Neurosci. 2010;167(2):323-328.

23. Holtzheimer PE, 3rd, Russo J, Claypoole KH, Roy-Byrne P, Avery DH. Shorter duration of depressive episode may predict response to repetitive transcranial magnetic stimulation. Depress Anxiety. 2004;19(1):24-30. 